\title{
Top-down technique for scaling to nano in silicon MEMS
}

Mustafa Yilmaz, Yasin Kilinc, Gokhan Nadar, Zuhal Tasdemir, Nicole Wollschläger, Werner Österle, Yusuf Leblebici, and B. Erdem Alaca

Citation: Journal of Vacuum Science \& Technology B 35, 022001 (2017); doi: 10.1116/1.4978047

View online: https://doi.org/10.1116/1.4978047

View Table of Contents: http://avs.scitation.org/toc/jvb/35/2

Published by the American Vacuum Society

\section{Articles you may be interested in}

Controlling color emission of InGaN/AIGaN nanowire light-emitting diodes grown by molecular beam epitaxy Journal of Vacuum Science \& Technology B, Nanotechnology and Microelectronics: Materials, Processing, Measurement, and Phenomena 35, 02B108 (2017); 10.1116/1.4977174

Surface preparation of freestanding GaN substrates for homoepitaxial GaN growth by rf-plasma MBE Journal of Vacuum Science \& Technology B, Nanotechnology and Microelectronics: Materials, Processing, Measurement, and Phenomena 35, 02B109 (2017); 10.1116/1.4977777

Engineering carrier lifetimes in type-II $\ln (\mathrm{Ga}) \mathrm{Sb} / \mathrm{InAs}$ mid-IR emitters

Journal of Vacuum Science \& Technology B, Nanotechnology and Microelectronics: Materials, Processing, Measurement, and Phenomena 35, 02B101 (2017); 10.1116/1.4972978

Sub-10 nm plasma nanopatterning of InGaAs with nearly vertical and smooth sidewalls for advanced $n$-fin field effect transistors on silicon

Journal of Vacuum Science \& Technology B, Nanotechnology and Microelectronics: Materials, Processing, Measurement, and Phenomena 35, 021206 (2017); 10.1116/1.4975796

Control of unintentional oxygen incorporation in $\mathrm{GaN}$

Journal of Vacuum Science \& Technology B, Nanotechnology and Microelectronics: Materials, Processing, Measurement, and Phenomena 35, 02B104 (2017); 10.1116/1.4975925

Oxidation and oxidative vapor-phase etching of few-layer $\mathrm{MoS}_{2}$ Journal of Vacuum Science \& Technology B, Nanotechnology and Microelectronics: Materials, Processing, Measurement, and Phenomena 35, 021203 (2017); 10.1116/1.4975144

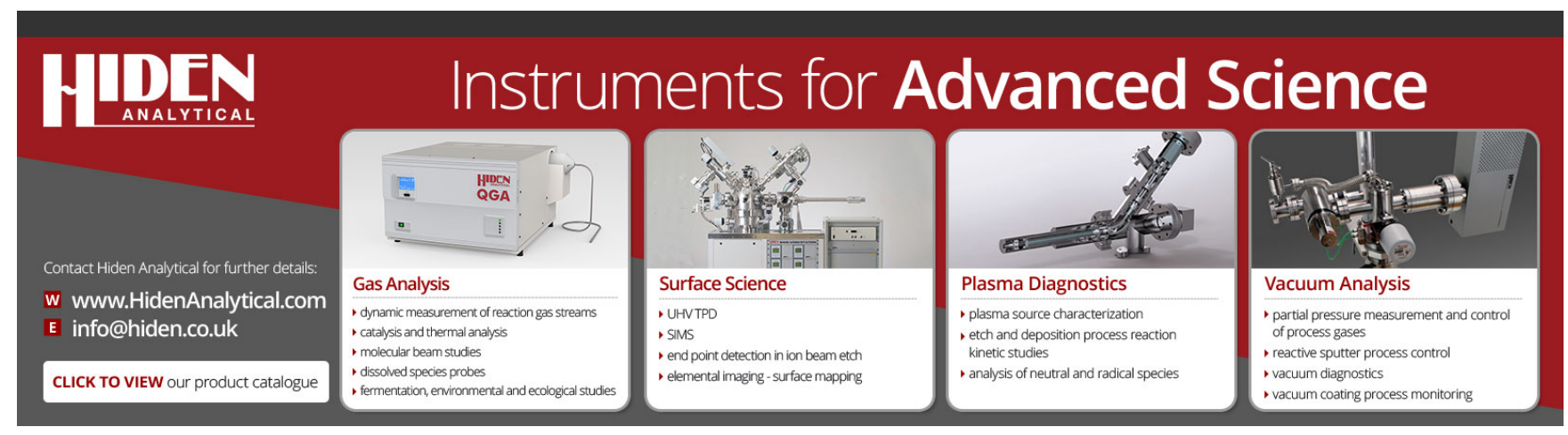




\title{
Top-down technique for scaling to nano in silicon MEMS
}

\author{
Mustafa Yilmaz, Yasin Kilinc, Gokhan Nadar, and Zuhal Tasdemir \\ Department of Mechanical Engineering, Koc University, Rumelifeneri Yolu, Sariyer 34450, Istanbul, Turkey \\ Nicole Wollschläger and Werner Österle \\ Bundesanstalt für Materialforschung und-prüfung (BAM), Unter den Eichen 87, D-12205 Berlin, Germany \\ Yusuf Leblebici \\ Microelectronic Systems Laboratory, École Polytechnique Fédérale de Lausanne, Bldg. ELD, \\ Station 11, CH-1015 Lausanne, Switzerland \\ B. Erdem Alaca ${ }^{\text {a) }}$ \\ Department of Mechanical Engineering, Koc University, Rumelifeneri Yolu, Sariyer 34450, Istanbul, Turkey \\ and Surface Science and Technology Center (KUYTAM), Koc University, Rumelifeneri Yolu, Sariyer 34450, \\ Istanbul, Turkey
}

(Received 8 December 2016; accepted 21 February 2017; published 8 March 2017)

\begin{abstract}
Nanoscale building blocks impart added functionalities to microelectromechanical systems (MEMS). The integration of silicon nanowires with MEMS-based sensors leading to miniaturization with improved sensitivity and higher noise immunity is one example highlighting the advantages of this multiscale approach. The accelerated pace of research in this area gives rise to an urgent need for batch-compatible solutions for scaling to nano. To address this challenge, a monolithic fabrication approach of silicon nanowires with 10- $\mu$ m-thick silicon-on-insulator (SOI) MEMS is developed in this work. A two-step Si etching approach is adopted, where the first step creates a shallow surface protrusion and the second step releases it in the form of a nanowire. It is during this second deep etching step that MEMS - with at least a 2-order-of-magnitude scale difference-is formed as well. The technique provides a pathway for preserving the lithographic resolution and transforming it into a very high mechanical precision in the assembly of micro- and nanoscales with an extreme topography. Validation of the success of integration is carried out via in situ actuation of MEMS inside an electron microscope loading the nanowire up to its fracture. The technique yields nanowires on the top surface of MEMS, thereby providing ease of access for the purposes of carrying out surface processes such as doping and contact formation as well as in situ observation. As the first study demonstrating such monolithic integration in thick SOI, the work presents a pathway for scaling down to nano for future MEMS combining multiple scales. (C) 2017 American Vacuum Society. [http://dx.doi.org/10.1116/1.4978047]
\end{abstract}

\section{INTRODUCTION}

Since the invention of the integrated circuit, miniaturization has been the driving factor for technological development. ${ }^{1}$ With device dimensions shrinking to quantum limits, it becomes increasingly challenging to maintain the pace of miniaturization while providing reliable operation. A number of candidate materials emerged in response to this challenge, among which one-dimensional nanostructures such as nanowires (NWs) constitute a major subset. ${ }^{2-4}$ Hence, the remarkable mechanical, electrical, and optical properties of NWs are under intense study. ${ }^{5-7}$

In addition to microelectronics, microelectromechanical systems (MEMS) benefit considerably from scaling to nano as well. New needs for semiconductor products are shaped by emerging areas such as internet of things, where the number of connected things is expected to reach $50 \times 10^{9}$ by the end of the year 2020. This development is to be facilitated by a massive on-chip integration effort of miniaturized sensors leading to products with more intelligence. ${ }^{8}$ In this regard, $\mathrm{Si}$ NWs gain a crucial importance due to their

${ }^{\text {a)} E l e c t r o n i c ~ m a i l: ~ e a l a c a @ k u . e d u . t r ~}$ potential for miniaturizing existing physical and biochemical sensors. ${ }^{9-12}$ This type of scaling into nano gives further rise to the need for a reliable electrical/mechanical interface. Based on a mature technology, MEMS is the leading candidate to assume the task of acting as such an interface and to provide a closely controlled environment for nanoscale sensor operation, for example, by imposing a well defined stress state. $^{13,14}$

Obtaining a deterministic assembly of MEMS with $\mathrm{Si}$ NWs constitutes a challenging task due to the considerable scale difference between the two entities. Both bottom-up and top-down fabrication methods are utilized to address this challenge. ${ }^{15}$ While the bottom-up approach is capable of providing very small (sub-10 nm) NWs with a well-defined chemistry-specifically when it is combined with electronbeam (e-beam) lithography for the definition of the growth location-the resulting NWs have a limited set of crystallographic orientations, and more importantly, the technique is not suitable for scaling up while abiding by the strict requirements of the deterministic assembly. ${ }^{16,17}$ On the other hand, the top-down approach offers a superior control on alignment and registry, while it is still capable of producing very small NWs. ${ }^{16}$ Hence, there is a consensus on the use of 
semiconductor processing techniques for scaling microscale systems to nano, ${ }^{8}$ while bottom-up products are successfully used in applications with less stringent requirements on integration. ${ }^{18}$

Integration of Si NWs and MEMS through the top-down approach is based on patterning and $\mathrm{Si}$ etching. On thin silicon-on-insulator (SOI) wafers, this directly translates into both NWs and MEMS having the same thickness. In one such example, where Si NWs are utilized as mechanical coupling elements for a flexural microresonator, all components uniformly exhibit a thickness of $275 \mathrm{~nm}$, which, at the same time, constitutes the SOI device layer thickness. ${ }^{19}$ Similarly, Si NW switch elements in another study have a thickness of $160 \mathrm{~nm}$, same as the thickness of all surrounding features. ${ }^{19}$ This approach based on thin SOI etching is adopted in almost all similar applications, ${ }^{20,21}$ as long as the need for incorporating an extreme topography does not surface.

However, there are instances, where an extreme topography cannot be omitted. In fact, there is a growing interest in combining Si NWs with much thicker MEMS, where-as opposed to the previous scenario-necessary processes remain highly involved and complicated. Incorporating such extreme topography differences opens up new possibilities such as piezoresistive sensors as miniature, multi-degree-offreedom, and low-power alternatives to capacitive MEMS with a high noise immunity. ${ }^{22,23}$ In such devices, Si NWs serve as highly sensitive and low-noise strain gauges attached to a set of much larger shuttles and flexures. Applications of this technology are recently demonstrated in the case of gyroscopes, ${ }^{24,25}$ magnetometers, ${ }^{13}$ microphones, ${ }^{26}$ and pressure sensors, ${ }^{14}$ where it is shown to lead to significant miniaturization. The principle of transduction is also well understood. ${ }^{27,28}$ Despite such operational advantages, the aforementioned architecture is achieved only as outlined in the previous paragraph, i.e., through working with thin SOI for the definition of the Si NW, on top of which a thick poly-Si layer is subsequently deposited. MEMS is then patterned and etched in this thick layer. ${ }^{22,23}$ The end result is a perfect example of the deterministic integration of different scales, where each component brings in its own share of enhanced functionality.

The results of this high-end approach are beyond the capability of any so-called "bottom-up" manufacturing, as both the orientation and location of Si NWs cannot be controlled to a precision necessary for a successful registry with subsequent lithography steps. However, the use of different $\mathrm{Si}$ layers for nano and microscale objects, in addition to the fact that the Si NW remains buried underneath the MEMS layer, raises a further need for an alternative, where both scales can be achieved in a thick SOI directly and Si NWs remain accessible for surface processes such as doping and contact formation.

One promising approach for fabricating Si NWs in bulk Si has recently been reported at a critical dimension (CD) of $20 \mathrm{~nm}^{29}$ This approach is almost a nanoscale counterpart of the single crystal reactive etching and metallization (SCREAM) process developed in the early 90 s for the fabrication of released microstructures in $\mathrm{Si}^{30}$ Here, a protrusion is created on the surface of the Si wafer by a shallow reactive ion etching (RIE) process. This protrusion is then coated with a protective conformal layer on its sidewalls. Si crystal is subsequently deep-etched further with an isotropic component, so that the Si protrusion is released under the protection of the conformal mask and assumes the form of a free-standing NW. An etch depth of $10 \mu \mathrm{m}$ has been demonstrated so far in bulk Si with room for further improvement. ${ }^{29}$

In this work, we explore the viability of adopting this integration approach for scaling MEMS to nano using a thick SOI wafer. A high level of mechanical precision is targeted such that a single Si NW attached to the tip of the shuttle of a MEMS comb-drive actuator will be perfectly aligned with respect to the force axis. As a result, the motion of the actuator tip will be directly translated into a uniaxial stress within the NW. Although appealing, adopting this process on a thick SOI is a challenging task and forms the backbone of this work. To our knowledge, no previous examples of such monolithic MEMS integration with a nanoscale building block exist spanning a 2 order-of-magnitude scale difference within the same Si crystal.

In the remainder of this paper, the integration process is introduced first followed by a description of the $\mathrm{Si}$ NW-MEMS assembly. Fabrication results are presented, and the paper is concluded with an in situ fracture experiment of the Si NW inside a scanning electron microscope (SEM).

\section{FABRICATION APPROACH TO SI NW-MEMS INTEGRATION}

The proposed approach for the monolithic integration of a Si NW with MEMS is demonstrated by fabricating the Si NW at the tip of the shuttle of a MEMS comb-drive actuator within a $10-\mu \mathrm{m}$-thick SOI device layer. With this architecture, the motion of the actuator is directly translated to stretching of the Si NW, which can, for example, be utilized as a uniaxial tensile testing platform for monolithic Si NWs. Although all patterning is carried out via e-beam lithography in this work, it should be emphasized that any high-resolution lithography can be utilized for this purpose, including mix-andmatch techniques.

A 4 in.-diameter, $\langle 100\rangle$ SOI wafer with a device layer thickness of $10 \pm 0.7 \mu \mathrm{m}$, a buried oxide (BOX) layer thickness of $1 \pm 0.05 \mu \mathrm{m}$, and a handle layer thickness of $380 \pm 10 \mu \mathrm{m}$ is used as the substrate. Si device layer of the SOI wafer has a resistivity of $1-10 \Omega \mathrm{cm}$. A device layer thickness of $10 \mu \mathrm{m}$ is chosen due to the relevance of the length scale for MEMS sensors and the etch depth achievement of previous work in bulk $\mathrm{Si}^{29}$

The fabrication procedure is summarized in Fig. 1. Determining the exact properties of the SOI wafer is important for calibrating process times and ensuring the best possible layout registration. First, the curvature of the wafer is determined via thin film stress measurement tool, Toho Technology FLX 2320-S. The exact thicknesses of both device and BOX layers are also measured via cross-sectional SEM inspection upon dicing the wafer prior to MEMS patterning. The first step of the fabrication [Fig. 1(a)] is the exposure of the Si NW 


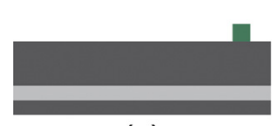

(a)

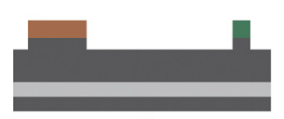

(d)

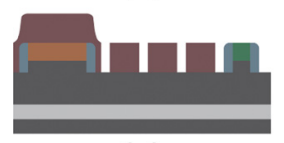

(g)

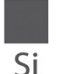
$\mathrm{BOX}$

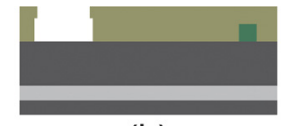

(b)

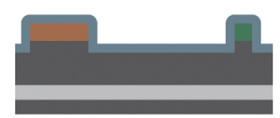

(e)

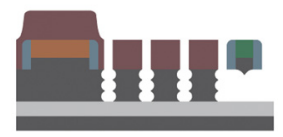

(h)

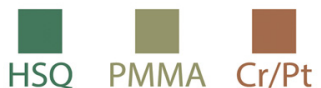

HSQ PMMA Cr/Pt

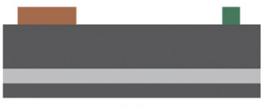

(c)

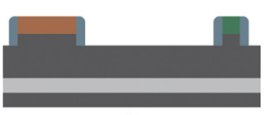

(f)

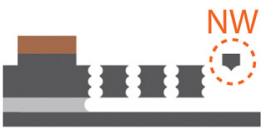

(i)
Si

SPTS APS dielectric dry etcher at $10^{\circ} \mathrm{C}$ [Fig. 1(f)]. Timing of this etch step is critical as excessive etch can remove the protective HSQ structure-which was transformed to a silica structure after electron beam irradiation ${ }^{31,32}$ - from the top surface of the Si NW. LTO etch rate is carefully measured as $4-4.5 \mathrm{~nm} / \mathrm{s}$. At this stage, Si surface is ready for the patterning of the MEMS actuator.

Prior to the patterning of the MEMS actuator, the wafer is diced into $12 \times 12 \mathrm{~mm}$ chips. Dicing is carried out for subsequent chip-level deep reactive ion etching (DRIE) optimization. MEMS patterning is performed using a 500-nm-thick positive-tone ZEP520A $100 \%$ e-beam resist by Zeon Corp with a dose of $225 \mu \mathrm{C} / \mathrm{cm}^{2}$ along with an accelerating voltage of $100 \mathrm{keV}$ [Fig. 1(g)]. After development in $n$-amylacetate solution for $1 \mathrm{~min}$ and $45 \mathrm{~s}$, DRIE of $\mathrm{Si}$ is conducted via Bosch process for $120 \mathrm{~s}$ in Alcatel AMS $200 \mathrm{SE}$ fluorine dry etcher [Fig. 1(h)]. As the etching is carried out at the single-chip level, a 2- $\mu$ m-thick AZ9260 positive resistcoated base wafer is used as the chip holder preventing the loading effect. AZ9260 is chosen as the base wafer coating because of its etch selectivity similar to that of ZEP520A $100 \%$ e-beam resist and relatively low cost.

Tuning of this second etch step is critical as it sets the extent of the undercut, which, in turn, leads to the release of the Si NW. A deep etch process with the alternating steps of plasma etching in $\mathrm{SF}_{6}$ (flow rate of $300 \mathrm{sccm}$ ) and passivation in $\mathrm{C}_{4} \mathrm{~F}_{8}$ (flow rate of $150 \mathrm{sccm}$ ) is developed with pulse times of 6.5 and $2 \mathrm{~s}$, respectively. Substrate temperature is kept at $30^{\circ} \mathrm{C}$. Pulsed low-frequency voltage biasing along with $600 \mathrm{~W}$ source generation power is utilized. These parameters are optimized for the release of defined Si NW geometries with proper undercuts. There is an upper limit to the Si NW width set by the extent of the DRIE undercut. If the Si NW is designed wider, release will not be complete. This might lead to Si whiskers underneath the Si NW or even an array of Si NWs as shown in our preliminary study on bulk $\mathrm{Si}^{29}$ Protection of the HSQ mask throughout the whole etch process has to be ensured as well, as HSQ acts as the only etch mask on the top surface of the Si NW, while side walls remain well protected under LTO.

Finally, oxide etching in $\mathrm{HF}$ vapor is carried out at $40^{\circ} \mathrm{C}$ [Fig. 1(i)]. This step removes all protection around the $\mathrm{Si}$ NW (both HSQ and LTO), while MEMS is also released through etching of the underlying BOX layer. Beyond this point, actuation of MEMS will directly translate into stretching of the Si NW. will later be necessary to mask $\mathrm{Si}$ in a series of further etch processes providing adequate protection during a final $10-\mu$ m-deep Si etch. Leading to a thicker HSQ protection, a shallower etch than $200 \mathrm{~nm}$ can sustain even harsher plasma conditions. A Si line of 200-nm thickness is thus obtained on the wafer surface that will later transform into a Si NW.

Fabrication of this Si NW protrusion is then completed with a conformal low temperature oxide (LTO) coating with a thickness of $100 \mathrm{~nm}$ through low-pressure chemical vapor deposition (LPCVD), carried out in a Centrotherm furnace at $425^{\circ} \mathrm{C}$ [Fig. 1(e)]. LTO is subsequently removed from lateral surfaces through dry etching with $\mathrm{He} / \mathrm{CHF}_{3}$ chemistry in

\section{RESULTS AND DISCUSSION}

Integration of a Si NW with a MEMS comb-drive actuator is demonstrated following the process flow given in Fig. 1. A representative sketch of the involved components - not drawn to scale-is given in Fig. 2 along with a list of actual dimensions. The width $(w)$ and length $(l)$ of the Si NW are determined by the HSQ pattern obtained at the end of the e-beam step of Fig. 1(a). However, the thickness of the Si NW $(t)$ depends on the depth of the shallow Si etch process of Fig. 1(d). MEMS dimensions ( $s, g$, and $b$ ) and 


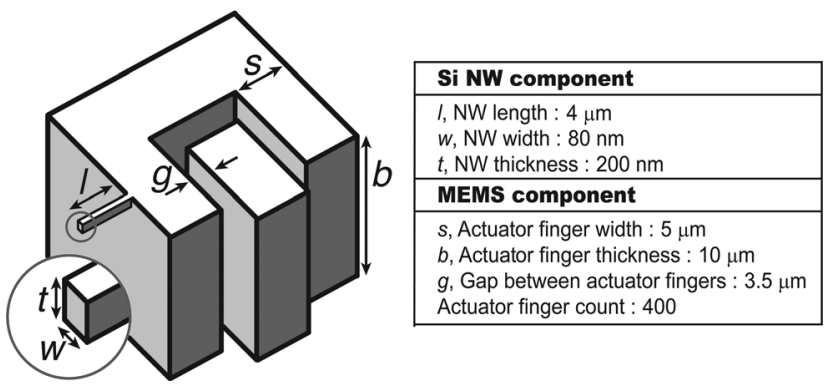

FIG. 2. Representative sketch and specifications for nano- and microscale components. Components are depicted in this symbolic architecture for demonstration purposes only and not drawn to scale.

actuator finger counts are determined based on the force requirements to induce fracture in the $\mathrm{Si} \mathrm{NW}$ upon actuating the system. Current design allows the application of a maximum force of $17.5 \mu \mathrm{N}$ for an actuation voltage of $40 \mathrm{~V}$. MEMS thickness $(b)$ is taken as SOI device layer thickness.

Achieving a 20-nm CD as proposed in Ref. 29, where no MEMS integration was involved, seems not to be possible in this work. This is a direct consequence of aspect-ratiodependent etching (ARDE), where prolonged etch times are necessary to remove $\mathrm{Si}$ at the bottom of narrow trenches between comb-drive digits [Fig. 1(h)]. While ARDE was not of any concern in the previous study on bulk $\mathrm{Si}^{29}$ in the current case with MEMS integration prolonged etching leads to the erosion of the HSQ mask. Nevertheless, a sub-100 nm CD can currently be demonstrated within a $10-\mu \mathrm{m}$ SOI device layer. $\mathrm{CD}$ can still be improved by utilizing an alternative, high-resolution and more resistant etch mask.

An image of the resulting $\mathrm{Si}$ NW-MEMS assembly is given in Fig. 3. Figure 3(a) depicts MEMS with comb-drives, springs, and a shuttle structure. The tip of the shuttle is directly linked to a Si NW as shown in the close-up view in Fig. 3(b) with the inset showing further details. Figure 3(c) provides a zoomed-in view of the Si NW-MEMS interface, highlighting the single-crystalline nature of the assembly. Inplane dimensions of the Si NW are also observed to be close to those listed in Fig. 2. To study its out-of-plane features, a detailed cross-sectional study is necessary.

The cross-section of the Si NW is studied by transmission electron microscopy (TEM). First, a focused ion beam (FIB) milling is carried out. The process starts with the coating of a thick Pt layer by the CVD system within the DualBeam FIB Quanta 3D FEG by FEI. After Si NWs are completely covered by $\mathrm{Pt}$, a small part of the Si NW close to its middle point is cut using the focused gallium ion beam at $30 \mathrm{kV}$. This slice is then transferred to a half-copper ring via an Omniprobe probing system. This is followed by further thinning of the slice with ion beam voltages decreasing from 30 to $2 \mathrm{kV}$ for final polishing. The resulting electrontransparent Si NW cross-section is finally investigated in TEM (JEM 2200FS by JEOL) using conventional and highresolution imaging to prove the crystalline character of the silicon wire.

Transmission electron micrograph obtained on the Si NW cross-section is given in Fig. 4. A trapezoidal cross-section
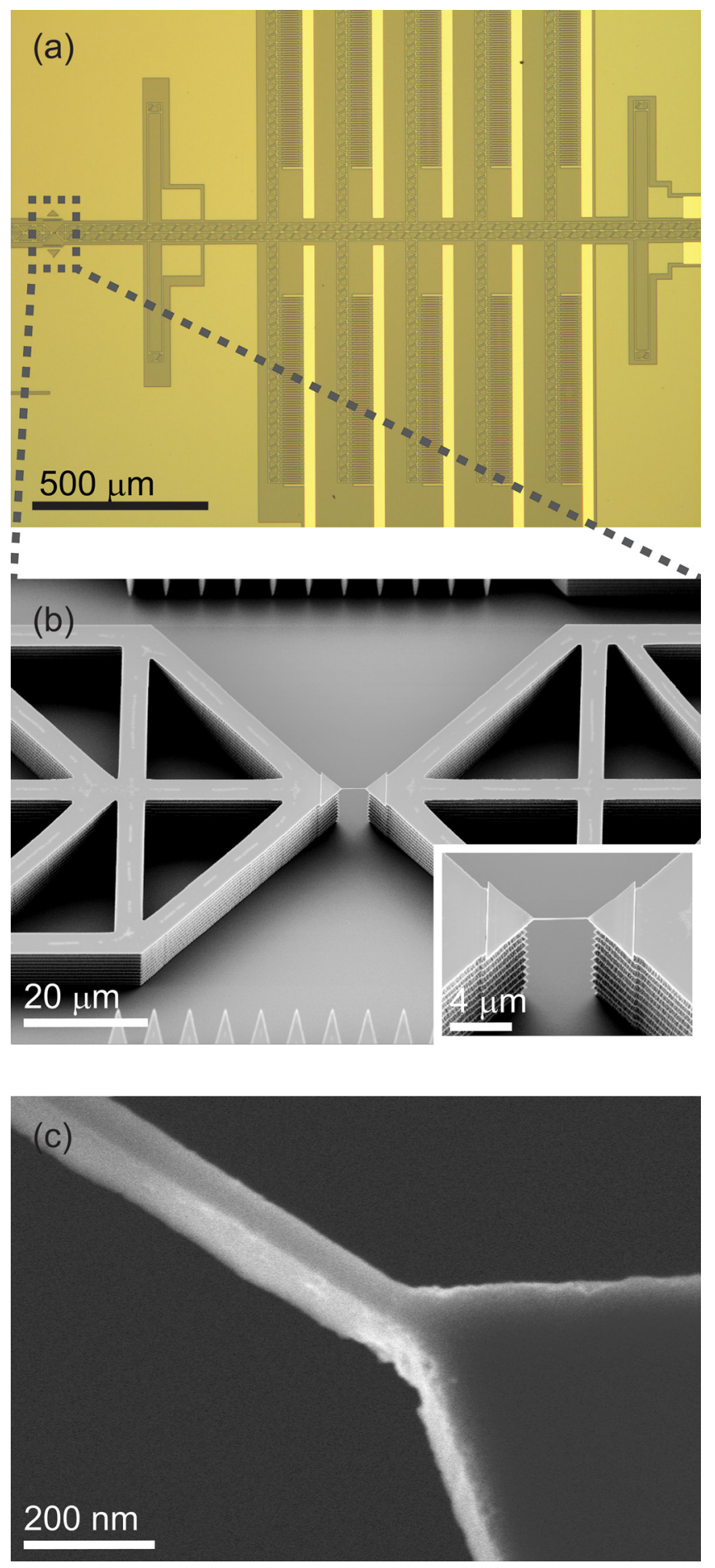

FIG. 3. (Color online) Si NW-MEMS assembly after fabrication. (a) An overall view of MEMS comb-drive actuator and Si NW in dashed box. (b) A close-up of the suspended Si NW with MEMS. The inset depicts a zoomedin view. (c) A close-up of the Si NW-MEMS interface.

is obtained with the width of $56 \mathrm{~nm}\left(\mathrm{w}_{1}\right)$ on the wafer surface increasing to $88 \mathrm{~nm}\left(\mathrm{w}_{2}\right)$ at the bottom of the Si NW. Inclined side walls are a byproduct of the RIE process of Fig. 1(d) and observed in our previous study on bulk $\mathrm{Si}$ as well. ${ }^{29,33}$ No whiskers or further Si NWs in the form of an array are observed, indicating that the extent of the DRIE undercut is adequate for complete release. ${ }^{29}$ High-resolution 

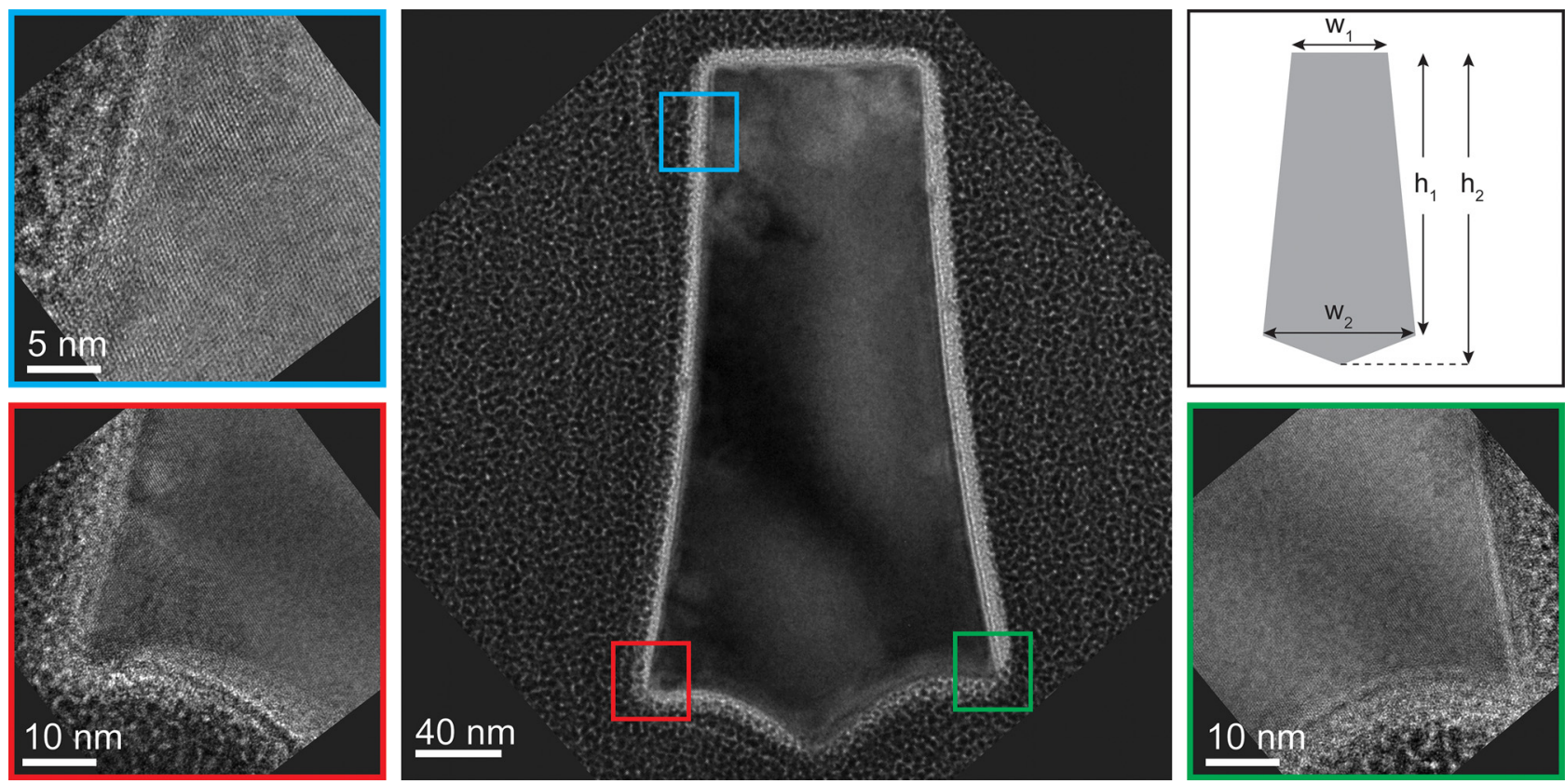

FIG. 4. (Color online) High-resolution TEM image of the trapezoidal Si NW cross-section. Insets depict close-up views from different zones of the Si NW. The schematic is provided as a guide for dimensional features.

close-up views of different zones of the cross-section are given in the insets of Fig. 4, where the native oxide coating and the crystalline structure of the Si NW core are visible. The Si NW thickness as defined by the etch depth of $200 \mathrm{~nm}$ of Fig. 1(d) is decreased to about $163 \mathrm{~nm}\left(\mathrm{~h}_{1}\right)$ due to Si etching along the unprotected bottom surface of the Si NW. Thickness through the center of the Si NW is $176 \mathrm{~nm}\left(\mathrm{~h}_{2}\right)$, which is caused by the scalloping effect of the DRIE process. Similar thinning was observed previously in bulk $\mathrm{Si}$ (Ref. 29) with slight differences due to DRIE parameters.

As a final remark, one should mention that as all processes after DRIE are carried out at the chip-level in this pilot production study, a reliable figure for yield cannot be reported. Although a series of successful devices are obtained in the current work, estimating yield remains the subject of future studies.

The deep etch process carried out on the SOI wafer is critical for the ultimate success of the Si NW-MEMS integration. A look into the alternative monolithic techniques at this point would be timely to highlight some of the associated challenges:

(1) An early example using oxide protection on the top and lateral surfaces of a nanoscale protrusion reports a very shallow release etch depth along with thickness uniformity issues due to the complications arising from the isotropic RIE process utilized. ${ }^{34}$ Variations of this isotropic release approach are reported recently with sidewall passivation achieved through $\mathrm{C}_{4} \mathrm{H}_{8} \cdot{ }^{35}$ As the Si NW thickness is significantly reduced during the release stage, the etch depths also remain confined to a maximum of $4 \mu \mathrm{m}$. This raises the need to start with much thicker surface protrusions, which reduces only to the order of $500 \mathrm{~nm}$ at the end of the release process.
(2) Instead of providing protection on top and lateral surfaces by HSQ and LTO, respectively, FIB-induced Ga doping is proposed. This process is shown to impart a very high selectivity during $\mathrm{Si}$ deep etch. With adequate undercut, doped $\mathrm{Si}$ can then be obtained in the form of a suspended NW. ${ }^{36,37}$ In this technique, NW thickness is determined only through adjusting the depth of the ion implantation. Furthermore, Si NW assumes an amorphous structure losing its single-crystal nature, and no possibility for other doping types exists. Annealing is later shown to help recover crystallinity. ${ }^{38}$

Following the dimensional characterization, resulting $\mathrm{Si}$ NW-MEMS assembly is placed inside an SEM chamber for actuation purposes. This facilitates in situ charging and actuation, where the generation of the electrostatic force is achieved by increasing the accumulated electron density on the comb drive actuator fingers. ${ }^{39}$ Figure 5 illustrates three stages of the in situ actuation experiment. Figure 5(a) shows the original configuration, where the Si NW is intact. In Fig. 5(b), MEMS actuation causes Si NW elongation leading to fracture. It should be emphasized that fracture was never observed in previous work with fixed $\mathrm{Si} \mathrm{NW}$ anchors. ${ }^{29,40}$ Finally, the contrast difference between fixed (left-hand side) and movable (right-hand side) fingers as depicted by the micrograph of Fig. 5(c) is an indication of the charging of the movable fingers leading to in situ actuation within SEM.

Although there are various techniques for Si NW integration with MEMS in a nonmonolithic fashion, they fall short of providing the demonstrated degree of parallel processing ability and dimensional control. Among those techniques, manipulation and mounting of separately fabricated Si NWs on MEMS is the leading one. ${ }^{4-43} \mathrm{Si}$ NWs can also be grown 

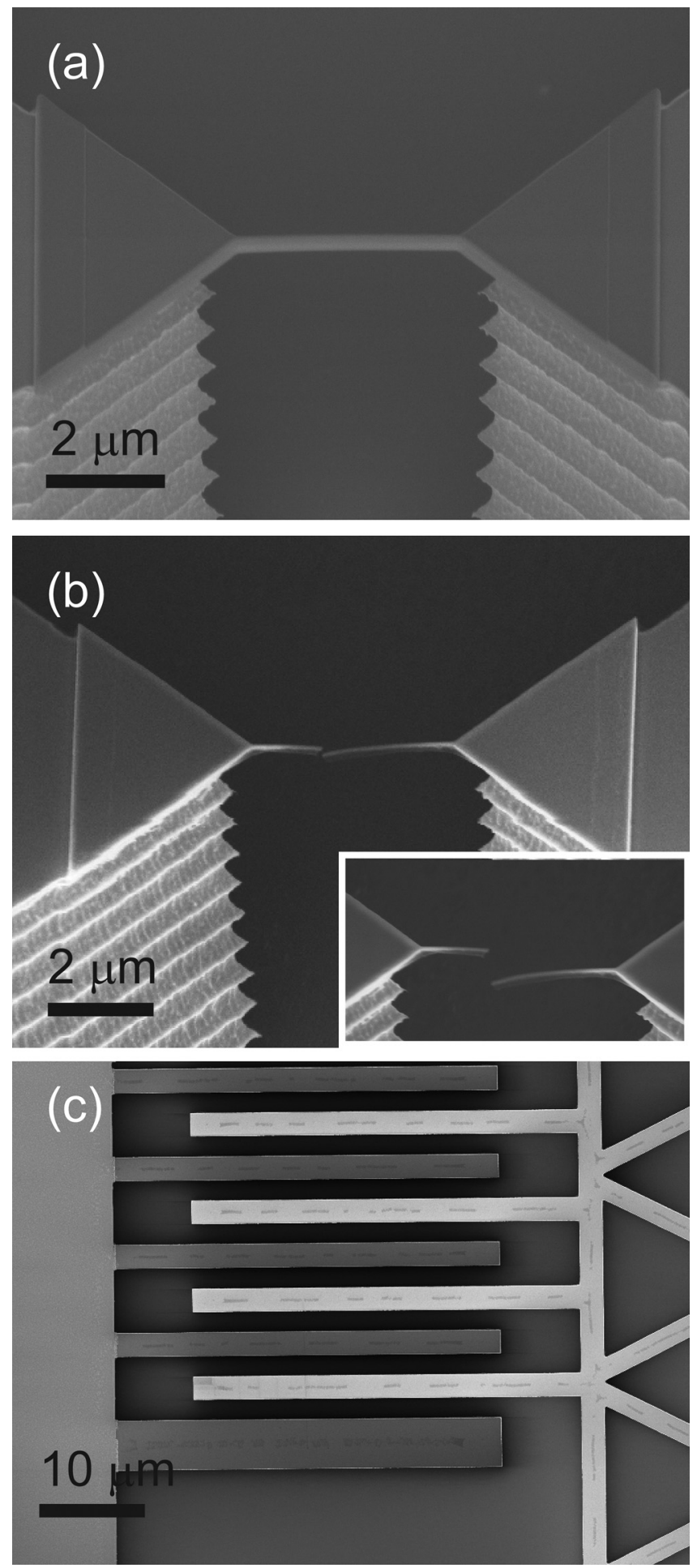

FIG. 5. Fracture of Si NW by in situ charging and the resulting MEMS actuation inside an SEM chamber. (a) Si NW before the fracture, (b) initial fracture of the Si NW, and (c) visible charging between MEMS comb-drive digits inside the SEM chamber.

on MEMS through VLS by the selective deposition of seed particles prior to growth. ${ }^{44,45}$ The only top-down approach that can address the shortcomings of the aforementioned techniques is based on the fabrication of the Si NW within the device layer of a thin SOI followed by the deposition of a thicker poly-Si layer for MEMS. ${ }^{22,23}$ However, the ability of fabricating Si NW and MEMS from the same crystal as proposed in the current work will lead to a very good level of structural integrity in the absence of any mechanical interface. In addition to the ease of processing, as only a single SOI is needed for achieving both micro- and nanoscales in a single device, having the Si NW close to the top surface will also facilitate selective doping and contact operations.

\section{CONCLUSION}

This study introduces a process technology for the monolithic integration of Si NWs with MEMS. The technology is demonstrated on conventional, 10- $\mu$ m-thick SOI substrates as opposed to alternative techniques involving a thin SOI for $\mathrm{Si}$ NW and a separate, thick Si coating for MEMS. This ability to work on thick SOI directly translates to simultaneous fabrication of the Si NW and MEMS using a two-step etch procedure resembling the SCREAM process. The first shallow Si etch creates a surface protrusion. This protrusion is protected by various etch masks against the second deep etch step, during which MEMS is formed and the protrusion is released, thanks to the isotropic component of the particular etch recipe. Fabrication is followed by the verification of the success of integration through an in situ fracture study of the Si NW.

In addition to the monolithic approach, the second most important advantage of the technique is the accessibility of the Si NW. The Si NW stands on the surface as opposed to being buried underneath the bulky MEMS, as it is the case with thin SOI processes followed by thick Si deposition. This feature brings about the ease of in situ observation along with the implementation of contact formation, doping, and other surface processes. There is also no fundamental limit to transferring the technology to even thicker SOI, for example, by replacing HSQ with a thicker negative-tone resist with a better etch resistance. Hence, this work paves the way to a range of possible multiscale devices with applications ranging from sensitive strain gauges in sensors to MEMS platforms for $\mathrm{Si}$ NW thermomechanical property measurements.

\section{ACKNOWLEDGMENTS}

This work was supported by Tubitak under Grant No. 112 E058 and Koc University-Istanbul Rotary Club Fundamental Research Seed Fund Program. M.Y., Y.K., and G.N. were supported by Tubitak 2214/A Fellowship. Z.T. acknowledges the Swiss Government Excellence Grant. Additionally, this project has received funding from the EMPIR programme cofinanced by the Participating States and from the European Union's Horizon 2020 research and innovation programme. The authors thank M. N. Esfahani for his help with fabrication.

${ }^{1}$ M. M. Waldrop, Nature 530, 144 (2016).

${ }^{2}$ L. Chen, F. Cai, U. Otuonye, and W. D. Lu, Nano Lett. 16, 420 (2016).

${ }^{3}$ M. De Marchi, D. Sacchetto, J. Zhang, S. Frache, P. E. Gaillardon, Y. Leblebici, and G. De Micheli, IEEE Trans. Nanotechnol. 13, 1029 (2014).

${ }^{4}$ N. Opondo, S. Ramadurgam, C. Yang, and S. Mohammadi, J. Vac. Sci. Technol., B 34, 011804 (2016).

${ }^{5}$ Y. Xia, P. Yang, Y. Sun, Y. Wu, B. Mayers, B. Gates, Y. Yin, F. Kim, and H. Yan, Adv. Mater. 15, 353 (2003). 
${ }^{6}$ F. Priolo, T. Gregorkiewicz, M. Galli, and T. F. Krauss, Nat. Nanotechnol. 9, 19 (2014).

${ }^{7}$ S. Dhara, E. J. Mele, and R. Agarwal, Science 349, 726 (2015).

${ }^{8}$ R. Singh, IEEE Electron Devices Society Newsletter, October (2015), Vol. 22, pp. 13-14.

${ }^{9}$ T. Mikolajick and W. M. Weber, Anisotropic Nanomaterials: Preparation, Properties, and Applications, edited by Q. Li (Springer International Publishing, Cham, 2015), pp. 1-25.

${ }^{10}$ P. Livi et al., Anal. Chem. 87, 9982 (2015).

${ }^{11}$ O. Leman, A. Nikas, H. Zhou, J. L. Lagos, B. J. Vinchhi, J. Hauer, G. Jourdan, and P. Rey, IEEE International Symposium on Circuits and Systems (ISCAS), Lisbon, Portugal, May (2015), pp. 666-669.

${ }^{12}$ S. Zhang, L. Lou, and C. Lee, Appl. Phys. Lett. 100, 023111 (2012).

${ }^{13}$ D. Ettelt, P. Rey, G. Jourdan, A. Walther, P. Robert, and J. Delamare, J. Microelectromech. Syst. 23, 324 (2014).

${ }^{14}$ Y. Deimerly, P. Robert, G. Jourdan, P. Rey, and T. Bourouina, paper presented at the 2013 Transducers Eurosensors XXVII: The 17th International Conference on Solid-State Sensors, Actuators and Microsystems (Transducers Eurosensors XXVII), 2013 (unpublished).

${ }^{15}$ B. E. Alaca, Int. Mater. Rev. 54, 245 (2009).

${ }^{16}$ M. M. Mirza, H. Zhou, P. Velha, X. Li, K. E. Docherty, A. Samarelli, G. Ternent, and D. J. Paul, J. Vac. Sci. Technol., B 30, 06FF02 (2012).

${ }^{17}$ M. Martin, S. Avertin, T. Chevolleau, F. Dhalluin, M. Ollivier, T. Baron, O. Joubert, and J. M. Hartmann, J. Vac. Sci. Technol., B 31, 041806 (2013).

${ }^{18}$ G. Gadea, A. Morata, J. D. Santos, D. Dávila, C. Calaza, M. Salleras, L. Fonseca, and A. Tarancón, Nanotechnology 26, 195302 (2015).

${ }^{19}$ N. Arellano, E. P. Quevy, J. Provine, R. Maboudian, and R. T. Howe, IEEE 21st International Conference on Micro Electro Mechanical Systems, 2008. MEMS 2008 (2008), pp. 721-724.

${ }^{20}$ L. Boodhoo, L. Crudgington, H. M. H. Chong, Y. Tsuchiya, Z. Moktadir, T. Hasegawa, and H. Mizuta, Microelectron. Eng. 145, 66 (2015).

${ }^{21}$ S. F. A. Rahman, N. A. Yusof, M. N. Hamidon, R. M. Zawawi, and U. Hashim, paper presented at the 2014 IEEE International Conference on Semiconductor Electronics (ICSE), 2014 (unpublished).

${ }^{22}$ P. Robert, V. Nguyen, S. Hentz, L. Duraffourg, G. Jourdan, J. Arcamone, and S. Harrisson, 2009 IEEE Sensors (2009), pp. 963-966.

${ }^{23}$ P. E. Allain, A. Bossboeuf, F. Parrain, S. Maaroufi, P. Coste, and A. Walther, paper presented at the 2012 IEEE 25th International Conference on Micro Electro Mechanical Systems (MEMS), 2012 (unpublished).

${ }^{24} \mathrm{~A}$. Walther et al., paper presented at the 2012 IEEE 25th International Conference on Micro Electro Mechanical Systems (MEMS), 2012 (unpublished).
${ }^{25}$ G. Langfelder, S. Dellea, A. Berthelot, P. Rey, A. Tocchio, and A. F. Longoni, J. Microelectromech. Syst. 24, 174 (2015).

${ }^{26}$ J. Czarny, A. Walther, B. Desloges, P. Robert, E. Redon, T. Verdot, K. Ege, C. Guianvarc'h, and G. L. Guyader, paper presented at the 2013 International Semiconductor Conference Dresden-Grenoble (ISCDG), Dresden, 2013 (unpublished).

${ }^{27}$ A. Koumela, D. Mercier, C. Dupré, G. Jourdan, C. Marcoux, E. Ollier, S. T. Purcell, and L. Duraffourg, Nanotechnology 22, 395701 (2011).

${ }^{28}$ G. Sberveglieri, V. Ferrari, G. Langfelder, S. Dellea, N. Aresi, and A. Longoni, Procedia Eng. 87, 1469 (2014).

${ }^{29}$ Z. Tasdemir, N. Wollschläger, W. Österle, Y. Leblebici, and B. E. Alaca, Nanotechnology 27, 095303 (2016).

${ }^{30}$ K. A. Shaw, Z. L. Zhang, and N. C. MacDonald, Sens. Actuators, A 40, 63 (1994).

${ }^{31}$ N. Takashi, S. Motoshi, K. Akihiko, S. Kiyotaka, and M. Katsutoshi, Jpn. J. Appl. Phys. 40, 6187 (2001).

${ }^{32}$ B. Kaleli, A. A. I. Aarnink, S. M. Smits, R. J. E. Hueting, R. A. M. Wolters, and J. Schmitz, STW ICT Conference (Technology Foundation STW, Utrecht, The Netherlands, 2010), pp. 105-108.

${ }^{33}$ H. V. Jansen, M. J. d. Boer, S. Unnikrishnan, M. C. Louwerse, and M. C. Elwenspoek, J. Micromech. Microeng. 19, 033001 (2009).

${ }^{34}$ A. N. Cleland and M. L. Roukes, Appl. Phys. Lett. 69, 2653 (1996).

${ }^{35}$ J. Wei, S. Magnani, and P. M. Sarro, Sens. Actuators, A 186, 80 (2012).

${ }^{36} \mathrm{~N}$. Chekurov, "Fabrication process development for silicon micro and nanosystems," Ph.D. thesis (Aalto University, Helsinki, Finland, 2011).

${ }^{37}$ M. D. Henry, M. Shearn, and A. Scherer, J. Vac. Sci. Technol., B 28, C6F26 (2010)

${ }^{38}$ J. Llobet, M. Sansa, M. Gerbolés, N. Mestres, J. Arbiol, X. Borrisé, and F. Pérez-Murano, Nanotechnology 25, 135302 (2014).

${ }^{39}$ A. L. Hogan, K. R. Ford, and I. R. Harvey, paper presented at the ASME 2012 International Mechanical Engineering Congress and Exposition, Houston, TX, 2012 (unpublished).

${ }^{40}$ Z. Tasdemir, O. Peric, D. Sacchetto, G. E. Fantner, Y. Leblebici, and B. E. Alaca, IEEE Nanotechnol. Express 1, 2 (2015).

${ }^{41}$ D. Zhang, J. M. Breguet, R. Clavel, V. Sivakov, S. Christiansen, and J. Michler, J. Microelectromech. Syst. 19, 663 (2010).

${ }^{42}$ T. Fujii, T. Namazu, K. Sudoh, S. Sakakihara, and S. Inoue, J. Eng. Mater. Technol. 135, 041002 (2013).

${ }^{43}$ R. A. Bernal, T. Filleter, J. G. Connell, K. Sohn, J. Huang, L. J. Lauhon, and H. D. Espinosa, Small 10, 725 (2014).

${ }^{44}$ Á. San Paulo, N. Arellano, J. A. Plaza, R. He, C. Carraro, R. Maboudian, R. T. Howe, J. Bokor, and P. Yang, Nano Lett. 7, 1100 (2007).

${ }^{45}$ W. Stefan, B. Emmerich, K. Shinya, I. Yoshitada, and L. Alois, Nanotechnology 25, 455705 (2014). 